\title{
Efficacy of clinical evaluations for COVID-19 on the front line
}

\author{
Lili L. Barsky ${ }^{1,2^{*}} \mathbb{D}$, Joseph E. Ebinger ${ }^{1}$, Mona Alotaibi ${ }^{3}$, Mohit Jain ${ }^{4}$, Sam Torbati ${ }^{5}$, Bradley T. Rosen ${ }^{6}$ and \\ Susan Cheng ${ }^{1,2}$
}

Keywords: Clinical assessment, Effectiveness, COVID-19

To the Editor:

In the midst of the COVID-19 pandemic, there remains limited availability of Food and Drug Administrationapproved tests for presence of the SARS-CoV-2 agent [1, $2]$. Even as testing capacity expands, optimization of resource utilization in the healthcare setting remains a significant priority $[3,4]$. Thus, the vast majority of front line work being done to evaluate for possible COVID-19 is highly dependent on the clinical assessment of a presenting patient's signs and symptoms. The extent to which current clinical assessments are effective, in the era of rapidly evolving local and professional guidelines, is not entirely clear.

We conducted a retrospective review of patients assessed for possible COVID-19 illness at our urban medical center in Los Angeles, California. The institutional review board deemed the study exempt. We carefully reviewed all clinical records to ascertain the provider's level of clinical suspicion for COVID-19 illness and compared these assessments with available results of SARSCoV-2 testing, in addition to longitudinal data on clinical outcomes. We found that the vast majority of patients ( $96 \%$ of $N=25$ ) clinically assessed to have a low probability of COVID-19 illness were subsequently confirmed to have either a negative SARS-CoV-2 test result or, in the absence of testing, clinical stability without any further concern for COVID-19 illness (Table 1). All clinical assessments were performed by a physician, with some (16\%) conducted by a nurse practitioner or physician assistant in conjunction with physician supervision.

In the absence of widespread readily available access to SARS-CoV-2 testing, clinical assessment is and will remain the standard of care for initially determining probability of COVID-19 illness and, in turn, appropriateness for receiving testing-especially in areas where testing availability is limited. This case series from an urban medical center suggests that despite the rapidly evolving body of knowledge around COVID-19 illness and its variable presentations among affected patients, clinical provider assessment of high versus low probability of active infection can be relatively reliable. This case series further supports the hypothesis that a well-informed clinical assessment, with or without concurrent access to rapid point-of-care SARS$\mathrm{CoV}-2$ testing, could be leveraged to more efficiently triage patients [5] —even those with medical comorbidities whose chronic illness burden may appear to pose a diagnostic challenge at the outset. In effect, a clinical evaluation that does not rely on viral testing results may be very accurate and substantially aid in ongoing efforts to conserve and appropriately prioritize the use of medical resources. Use of sound clinical judgment can also facilitate consideration of alternative diagnostic explanations.

\footnotetext{
* Correspondence: oodlesoflili@gmail.com

${ }^{1}$ Department of Cardiology, Smidt Heart Institute, Cedars-Sinai Medical

Center, 127 S. San Vicente Boulevard, Los Angeles, CA 90048, USA

${ }^{2}$ Barbra Streisand Women's Heart Center, Cedars-Sinai Medical Center, $127 \mathrm{~S}$.

San Vicente Boulevard, Los Angeles, CA 90048, USA

Full list of author information is available at the end of the article
} 


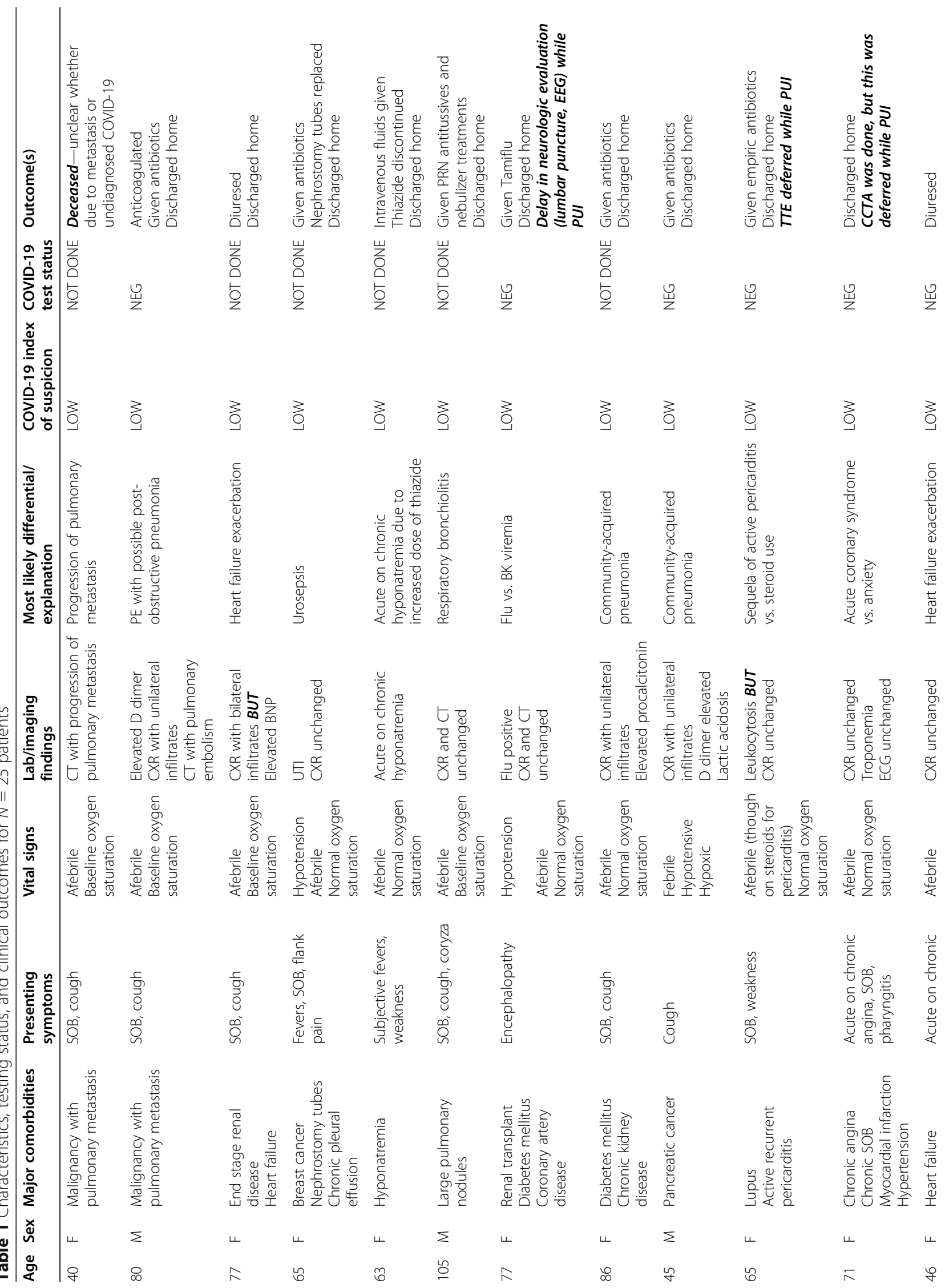




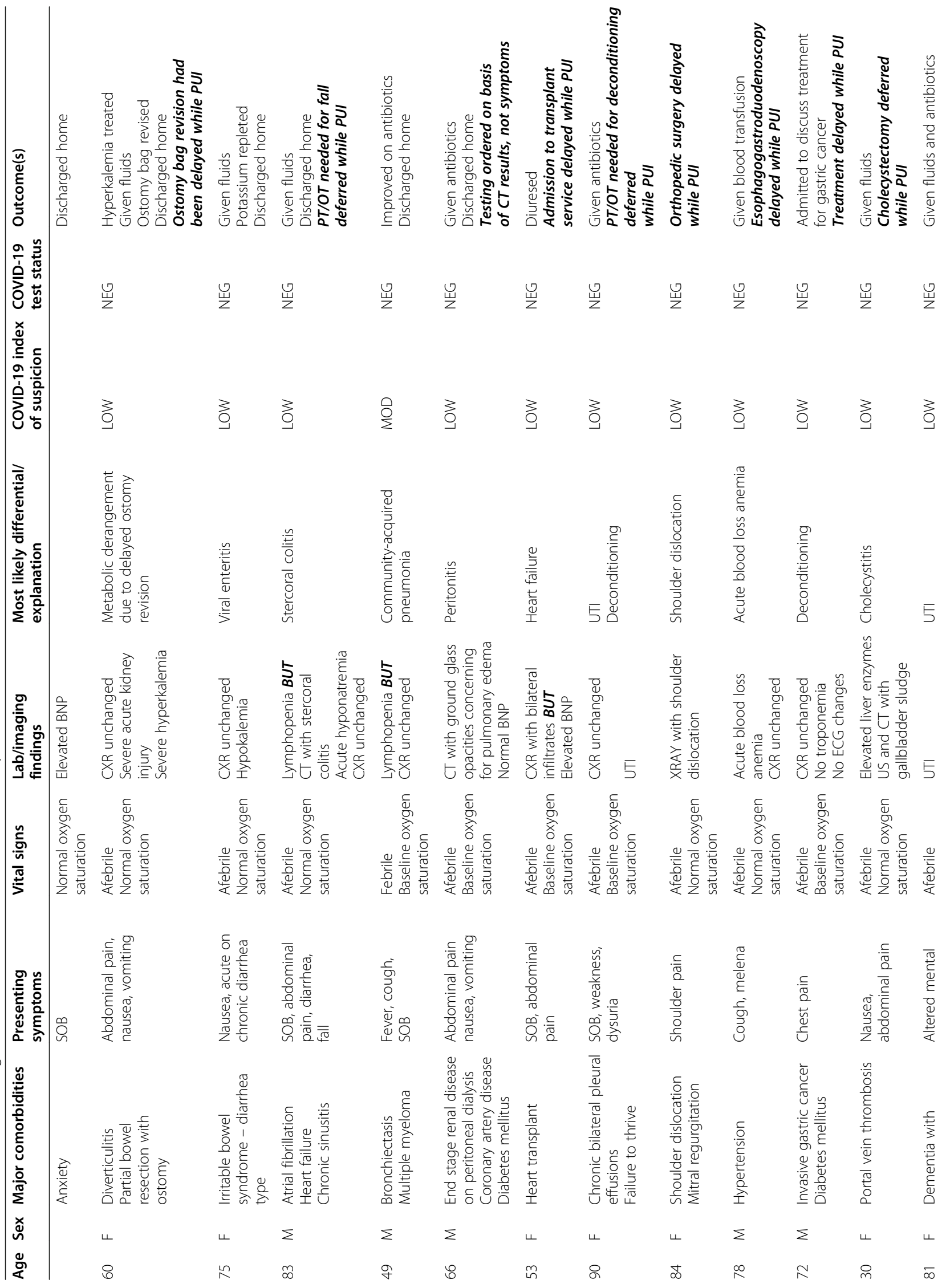




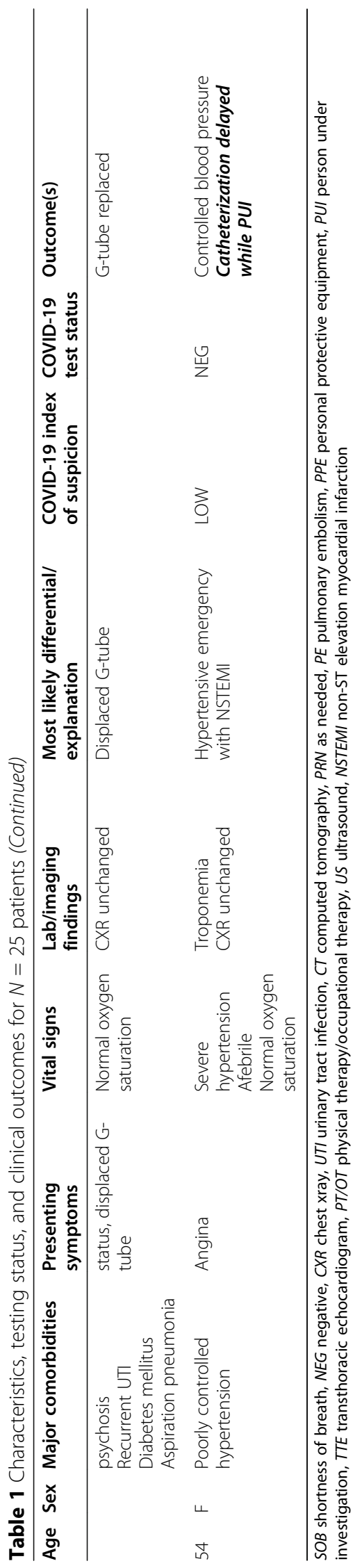




\section{Acknowledgements}

Not applicable.

\section{Authors' contributions}

LB conceived of the study concept and design, helped acquire the data from the emergency department and inpatient setting, performed the analysis and interpretation of the data, and drafted the manuscript. JE provided critical revision of the manuscript for important intellectual content and provided statistical expertise regarding the analysis. MA and $\mathrm{MJ}$ also critically revised the manuscript for important intellectual content. ST and BR helped acquire the data from the emergency department and inpatient setting and also critically revised the manuscript for important intellectual content. SC helped formulate the study concept and design, helped with analysis and interpretation of the data, provided statistical expertise, and critically revised the manuscript for important intellectual content. All authors read and approved the final manuscript.

\section{Funding}

This work was supported in part by Cedars Sinai Medical Center, the Erika J Glazer Family Foundation, and NIH/NCl grant U54-CA260591.

\section{Availability of data and materials}

All data generated or analyzed during this study are included in this published article.

\section{Ethics approval and consent to participate}

The institutional review board deemed the study exempt.

\section{Consent for publication}

Not applicable.

\section{Competing interests}

The authors declare that they have no competing interests.

\section{Author details}

'Department of Cardiology, Smidt Heart Institute, Cedars-Sinai Medical Center, 127 S. San Vicente Boulevard, Los Angeles, CA 90048, USA. ${ }^{2}$ Barbra Streisand Women's Heart Center, Cedars-Sinai Medical Center, 127 S. San Vicente Boulevard, Los Angeles, CA 90048, USA. ${ }^{3}$ Department of Pulmonology, University of California San Diego, 200 W. Arbor Drive, San Diego, CA 92103, USA. ${ }^{4}$ Department of Medicine and Pharmacology, University of California San Diego, 200 W. Arbor Drive, San Diego, CA 92103, USA. ${ }^{5}$ Department of Emergency Medicine, Cedars-Sinai Medical Center 8700 Beverly Boulevard, Los Angeles, CA 90048, USA. ${ }^{\text {In }}$ matient Specialty Program, Cedars-Sinai Medical Center, 8700 Beverly Boulevard, Los Angeles, CA 90048, USA.

Received: 18 June 2020 Accepted: 26 October 2020

Published online: 07 November 2020

\section{References}

1. U.S. Food \& Drug Administration. Drug Shortages Response| COVID-19. https://www.fda.gov/drugs/coronavirus-covid-19-drugs/drug-shortagesresponse-covid-19. Accessed 18 April 2020.

2. Jamil S, Mark N, Carlos G, Dela Cruz CS, Gross J, Pasnick S. Diagnosis and management of COVID-19 disease. ATS public health information series. Am J Respr Crit Care. 2020;201:19-22.

3. Prachand V, Milner R, Angelos P. Medically necessary, time-sensitive procedures: scoring system to ethically and efficiently manage resource scarcity and provider risk during the COVID-19 pandemic. J Am Coll Surg. 2020;231(2):281. https://doi.org/10.1016/j.jamcollsurg.2020.04.011.

4. Glauser W. Proposed protocol to keep COVID-19 out of hospitals. CMAJ. 2020;192(10):E264. https://doi.org/10.1503/cmaj.1095852.

5. McCullough LB. In response to COVID-19 pandemic physicians already know what to do. Am J Bioeth. 2020;20(7):9. https://doi.org/10.1080/ 15265161.2020.1754100.

\section{Publisher's Note}

Springer Nature remains neutral with regard to jurisdictional claims in published maps and institutional affiliations. 